\title{
Zum Stoffwechsel des Glukosamins in Ascitestumorzellen
}

\author{
III. Mitteilung: Stoffwechselwirkungen von Glukosaminderivaten
}

Von Th. Günther, M. Wenzel und T. Eberhard

Ails dem Ply'siologisch-Chemischen Institut der Freien Universität Berlin (Direktor: Prof. Dr. med. Dr. rer. nat. E. Schïtte)

(Der Schriftleitung zugegangen am 24. September 1964)

\begin{abstract}
Bei Inkubation von Ascitestumorzellen mit $\beta$-glukosaminidisch verbundenen Oligomeren des Glukosamins wird die endogene Atmung allmählich zunehmend gehemmt. Nach etwa 20-40 Minuten ist die endogene Atmung im selben Maße gehemmt wie nach Zugabe von Glukose oder Glukosamin. Die Oligomeren des Glukosamins werden hydrolysiert. Das entstehende Glukosamin wird von den Ascitestumorzellen resorbiert. Die Oligomeren des N-Acetylglukosamins haben keinen Einfluß auf die endogene Atmung von Ascitestumorzellen und werden, wie mit $\mathrm{T}$-markierter $\mathrm{N}$-Acetylchitotriose gezeigt wurde, auch nicht gespalten. - Die intrazelluläre $\mathrm{PO}_{4}$-Konzentration nimmt nach Zugabe von Glukosamin stark, nach Zugabe von Glukose wenig und nach Zugabe von Oligosacchariden des Glukosamins überhaupt nicht ab.

Hyaluronsäure wird, wie aus dem Verhalten des Reduktionswertes und aus Versuchen mit T-markierter Hyaluronsäure folgt, von Ascitestumorzellen hydrolysiert. Chondroitinschwefelsäure dagegen wird von Ascitestumorzellen wahrscheinlich nicht gespalten.

The respiration of ascites tumour cells shows a gradually increasing inhibition on incubation with $\beta$-linked oligomeric glucoseamine. After 20-40 min., the endogenous respiration is inhibited to the same extent as after the addition of glucose or glucoseamine. The oligoglucoseamine is hydrolysed and the resulting glucoseamine is absorbed by the ascites tumour cells. Oligomeric $\mathrm{N}$-acetylglucoseamine has no influence on the endogenous respiration of ascites tumour cells and, as shown with tritiated $\mathrm{N}$-acetylchitotriose, it is not hydrolysed. After the addition of glucoseamine, the intracellular $\mathrm{PO}_{4}$-concentration decreases markedly; glucose causes a smaller decrease; oligoglucoseamine has no effect. Hyaluronic acid is hydrolysed by ascites tumour cells, as shown by the change in reduction value and in experiments with tritiated hyaluronic acid. Chondroitin sulphate, however, is probably not hydrolysed.
\end{abstract}

In früheren Mitteilungen $(1,2,3)$ haben wir den Stoffwechsel des Glukosamins in Ascitestumorzellen beschrieben. Glukosamin ist Baustein der Mucopolysaccharide des Bindegewebes. Wir haben deshalb in der vorliegenden Arbeit die Untersuchungen auf die Stoffwechselwirkungen von polymeren Glukosaminderivaten ausgedehnt, um einen Einblick in die Beziehungen $z$ wischen Mucopolysacchariden und Tumorwachstum zu erhalten. $\mathrm{Da}$ andere Bausteine von Mucopolysacchariden wie NAcetylglukosamin, Galaktosamin, N-Acetylgalaktosamin und Glukuronsäure den Tumorstoffwechsel nicht verändern, können nur Glukosamin oder Glukosamin enthaltende Mucopolysaccharide (4) einen Einfluß auf Tumorzellen ausüben. Wir untersuchten deshalb als Modellsubstanzen $\beta$-glukosaminidisch verknüpfte Oligomere des Glukosamins und N-Acetylglukosamins sowie Hyaluronsäure als physiologisch vorkommendes $\beta$-glukosaminidisch gebundenes Glukosaminderivat.

\section{Methodik}

Für die Versuche verwendeten wir Ehrlich-Mäuseascitestumorzellen (ELD, Stockholm). Die Zellen wurden zwischen dem 7. und 10. Tag nach intraperitonealer Inokulation entnommen und sofort in Krebs-Ringer-Lösung blutfrei gewaschen. Anschließend wurden die Zellen bei $37^{\circ}$ in Krebs-Ringer-Bikarbonat, KrebsRinger-Phosphat oder in Ascitesserum, das. $10 \mathrm{Min}$. bei $56^{\circ}$ inaktiviert worden war, inkubiert.

Der Sauerstoffverbrauch wurde manometrisch nach der direkten Methode mit Luft als Gasphase bestimmt. Die Substratkonzentration betrug dabei jeweils $2 \mathrm{mg} / \mathrm{ml}$.

Der Zellgehalt der Suspensionen wuirde durch Zentrifugieren ( 5 Min. bei $4000 \mathrm{~g}$ ) in geeichten Eiweißrelationsröhrchen nach KAFKA bestimmt. Zur Trennung von Zellen und Inkubations- medium wurde die Zellsuspension $4 \mathrm{Min}$. bei etwa $4000 \mathrm{~g}$ zentrifugiert.

Der Orthophosphat-Gehalt der Zellen wurde im TCE-Extrakt der Zellen nach FISKE und SUBbarow ermittelt (5).

Die Spaltung der inaktiven Saccharide und der inaktiven Hyaluronsäure bestimmten wir mit Hilfe des Reduktionswertes. Das durch Zentrifugieren abgetrennte Inkubationsmedium (Krebs-RingerLösung) wurde im Verhältnis 1:1 mit 10-proz. Trichloressigsäure enteiweißt und anschließend mit $\mathrm{NaHCO}_{3}$ neutralisiert. Der Reduktionswert wurde nach PARK-JoHNson bestimmt (6).

\section{Substrate}

Als Substrate dienten Glukosamin $(\mathrm{GA})^{1}$ ) und seine $\beta$-glukosaminidisch verbundenen Oligomeren $(G A)_{2},(G A)_{3},(G A)_{4}$, sowie die entsprechenden $\mathrm{N}$-Acetylierungsprodukte $\mathrm{N}$-acetyl-GA, Di$(\mathrm{N} \text {-acetyl)-(GA) } 2 \text {, Tri-(N-acetyl)-(GA) })_{3}$ Tetra-(N-acetyl)-(GA) Die nicht-acetylierten Saccharide wurden aus Krebsschalen durch $\mathrm{HCl}$-Hydrolyse und anschließende Fraktionierung über eine mit Dowex 50 gefüllte Säule (7) dargestellt. Zur Darstellung der acetylierten Substanzen wurden die nicht-acetylierten Saccharide anschließend an den Aminogruppen acetyliert (8). K-Hyaluronat und Na-Chondroitinsulfat ${ }^{2}$ ).

\section{Herstellung T-markierter Substrate}

Tri-(N-acetyl)-chitotriose-[T] wưrde durch WrLZBACEI-Markierung hergestellt und durch Umfällen und Papierchromatographie gereinigt (9).

Hyaluronsäure wurde ebenfalls nach WILZBACH markiert. In einer einfachen und deshalb besonders sicheren Apparatur (10) wurden $149 \mathrm{mg}$ Hyaluronsäure mit 5 Curie $T_{2} 4$ Tage bei $0^{\circ}$ inkubiert. Anschließend wurde die markierte Hyaluronsäure zur Entfernung

1) Abkürzungen: $\mathrm{GA}=$ Glukosamin; $(\mathrm{GA})_{2}=$ Chitobiose; $(\mathrm{GA})_{3}=$ Chitotriose $\left.(7) ; \mathrm{GA}\right)_{4}=$ Chitotetraose (7).

2) Wir danken Herrn Dr. H. Grbian (Schering AG, Berlin) für die großzügige Überlassung von K-Hyaluronat und Na-Chondroitinsulfat. 
des labilen Tritiums mehrmals in Wasser gelöst und gefriergetrocknet.

Um das Produkt zu reinigen, lösten wir die markierte Hyaluronsäure in Wasser und fällten mit einer gesättigten Lösung von Kaliumacctat in A thanol. Das gefällte Produkt wurde dreimal mit Alkohol und zweimal mit Ather gewaschen und anschlicßend über $\mathrm{P}_{2} \mathrm{O}_{5}$ getrocknet (1. Schritt). Danach wurde die Hyaluronsäurc durch Mehrfachchromatographic (6 mal aufsteigend auf Schleicher und Schüll 2043b in Pyridin/n-Pentanol-(2)/Wasser 18:8:7\} von niedermolekularen Beimengungen befreit (2. Schritt). Weiterhin wurde die Reinigungsoperation durch Pällung der vom Papier eluierten Hyaluronsäure $2 \mathrm{mal}$ wiederholt (3. und 4. Schritt). Nach jedem Schritt wurde die spezifische Aktivität der Hyaluronsäure-[T] bei einer Schichtdicke von $1 \mu \mathrm{g} / \mathrm{cm}^{2}$ auf dem Schälchen nach RyDuERG (11) im Methanstrom-Zählrohr FH 407 gemessen. Zählausbeute: $43,5 \%$; Fehler: $\pm 6 \%$. Nach den einzelnen Reinigungsschritten wurden folgende spezifischen Aktivitäten erhalten:

\begin{tabular}{lc}
\hline & Spez. Aktivität in $[\mu \mathrm{c} / \mathrm{mg}]$ \\
\hline 1. Schritt & 0,63 \\
2. Schritt & 0,44 \\
3. Schritt & 0,46 \\
4. Schritt & 0,45 \\
\hline
\end{tabular}

Man erhält also eine von niedermolekularen Verunreinigungen freie Hyaluronsäurc-[T] der spezifischen Aktivität $0,45 \mu \mathrm{c} / \mathrm{mg}$.

\section{Aktive Vrersuche}

Um entscheiden zu können, ob Oligomere des $\mathrm{N}$-acetylglukosamins gespalten werden, wurde T-markierte Tri-(N-acetyl)-(GA) $)_{3}$ mit Ascitestumorzellen in Krebs-Ringer-Hydrogenkarbonat-Lösung $60 \mathrm{Min}$. bei $37^{\circ}$ geschüttelt.

Das Inkubationsmedium und der Trichloressigsäure-Extrakt der Zellen wurden dreifach (9) chromatographiert.

Die T-markierte Hyaluronsäure wurde wie in Abbildung 5 angegeben mit Trockenpulver von Ascitestumorzellen bei $\mathrm{pH}=5,5$ inkubiert.

Die Aktivitätsverteilung auf den Chromatogrammen wurde mit einem Berthold-Doppelzählrohr (10) gemessen, das mit dem Papierchromatographen FH 452 und Zählgerät FH 49 mit Linienschreiber verbunden war (2).

\section{Ergebnisse}

\section{Atmungshemmung nach Zugabe verschiedener Substrate}

Werden Ascitestumorzellen mit Glukose oder Glukosamin inkubiert, so wird mit der manometrischen Methode eine sofortige Hemmung der endogenen Atmung registriert. Mit $(G A)_{2},(G A)_{3}$ und $(G A)_{4}$ dagegen erfolgt eine sich nur allmählich auswirkende Hemmung der endogenen Atmung (Abb. 1). Nach etwa 20-40 Minuten ist die Atmung im gleichen Ausmaße gehemmt wie nach Zugabe von Glukose und Glukosamin. Die Zeitspanne bis zum Erreichen der maximalen Atmungshemmung hängt vom Zellgehalt der Zellsuspension ab. Bei hohem Zellgehalt wird die Atmung schneller gehemmt als bei geringen Zellkonzentrationen. Dieses Verhalten fanden wir nur mit den nicht-acetylierten Verbindungen. Die acetylierten Saccharide beeinflussen, wie Tabelle 1 zeigt, die endogene Atmung von Ascitestumorzellen nicht.

Zur näheren Charakterisierung dieses Effektes wurde geprüft, ob die Hemmung der endogenen Atmung reversibel ist. Hierzu wurden die Zellen so lange in- kubiert, bis die endogene Atmung durch die freien Saccharide in gleichem Ausmaß wie durch Glukose und Glukosamin gehemmt war. Sodann wurden die Substrate durch zweimaliges schonendes Waschen entfernt und die Zellen ohne Substrat reinkubiert. Aus Tabelle 2 folgt, daß die Atmungshemmung durch Glukose vollständig reversibel ist, während die Atmungshemmung durch Glukosamin und seine Oligomeren nicht oder nur zu einem geringen Teil reversibel ist.

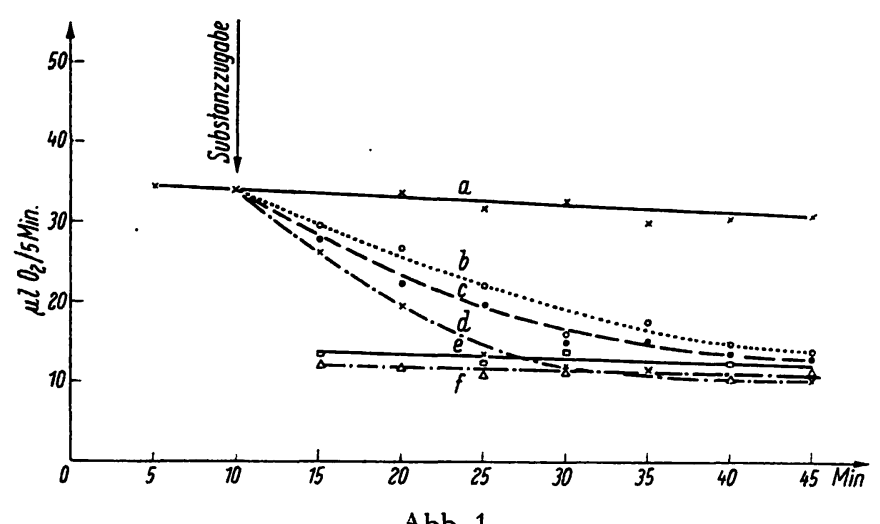

Hemmung der endogenen Atmung durch Oligosaccharide des Glukosamins
a) Kontrolle ohne Substrat
b) $(\mathrm{GA})_{2}$
c) $(\mathrm{GA})_{4}$
d) $(\mathrm{GA})_{3}$
e) Glukose
f) Glukosamin

„Pfeil“: Einschwenken der Substrate.

Die Zellen (Zellkonzentration: 8,5 Vol. \%) wurden in $2,2 \mathrm{~m} /$ Krebs-Ringer-Phosphat-Lösung $\mathrm{pH}=7,4 \mathrm{mit}$ Substrat $(2 \mathrm{mg} / \mathrm{m} /)$ bei $37^{\circ} \mathrm{im}$ Warburgapparat inkubiert.

Tab. 1

Atmung von Ascitestumorzellen

In $2,2 \mathrm{~m} /$ Krebs-Ringer-Phosphat $(\mathrm{pH}=7,4)$ befanden sich $150 \mu \mathrm{l}$ $(6,8 \mathrm{Vol} . \%)$ Zellen. Die Atmung wurde zunächst 20 Minuten ohne Substrat und dann nach Zugabe von Substrat gemessen. (Meßdaten für den $\mathrm{O}_{2}$-Verbrauch s. analogen Versuch in Abb. 1)

\begin{tabular}{lcc}
\hline $\begin{array}{c}\text { Substrat } \\
(2 \mathrm{mg} / \mathrm{m} /)\end{array}$ & \multicolumn{2}{c}{$\begin{array}{c}\text { Atmung } \\
\left(\mu / \mathrm{O}_{2} / \text { Std. } \mu / \text { Zellen }\right)\end{array}$} \\
\hline ohne Substrat & 1,81 & 1,78 \\
N-acetyl-GA & 1,73 & 1,71 \\
$\mathrm{~N}$-acetyl-(GA) $)_{2}$ & 1,74 & 1,68 \\
N-acetyl-(GA) & 1,69 & 1,65 \\
N-acetyl-(GA) & 1,76 & 1,69 \\
Glukose & 1,72 & 0,65
\end{tabular}

Tab. 2

Reversibilität der Atmungshemmung

In $2,2 \mathrm{~m} /$ Krebs-Ringer-Phosphat $(\mathrm{pH}=7,4)$ befanden sich $127 \mu l$ Zellen. Die Ansätze wurden 25 Minuten bei $37^{\circ}$ mit Substraten (jeweils $2 \mathrm{mg} / \mathrm{ml}$ ) bis zur maximalen Hemmung der Atmung vorinkubiert, sodann zweimal mit Krebs-Ringer-Phosphat-Lösung gewaschen und ohne Substrat reinkubiert.

\begin{tabular}{cc}
\hline Vorinkubation & $\begin{array}{c}\text { Atmung nach Auswaschen des Substrates } \\
\left(\mu / \mathrm{O}_{2} / \text { Std. } \cdot \mu / \text { Zellen }\right)\end{array}$ \\
\hline ohne Substrat & 1,89 \\
Glukose & 1,84 \\
$\mathrm{GA}$ & 1,37 \\
$(\mathrm{GA})_{2}$ & 1,23 \\
$(\mathrm{GA})_{3}$ & 0,95 \\
$(\mathrm{GA})_{4}$ & 1,52
\end{tabular}




\section{Spaltung der Oligosaccbaride}

Die eben beschriebenen Versuche lassen vermuten, daß die Atmungshemmung der Saccharide durch eine $\mathrm{Ab}$ spaltung von Glukosamin bedingt ist. Es wurde deshalb deren Spaltbarkeit untersucht. Das Ergebnis zeigt Tabelle 3. Aus der gleichen Zunahme des Reduktionswertes folgt, daß alle untersuchten nicht-acetylierten Saccharide im gleichen Ausmaß von Ascitestumorzellen gespalten werden. Aus der in Tabelle 4 aufgeführten Abnahme des Hexosaminwertes ergibt sich weiterhin, daß ein Teil des abgespaltenen Glukosamins in die Zellen eingedrungen ist. Daß Glukosamin von Ascitestumorzellen aufgenommen und intrazellulär abgebaut wird, konnten wir schon früher nachweisen (2). Die

Tab. 3

Spaltung von Oligosacchariden des Glukosamins durch Ascitestumorzellen

In $2,5 \mathrm{~m} l$ Krebs-Ringer-Bikarbonat-Lösung befanden sich $152 \mu l$ Zellen und jeweils $5 \mathrm{mg}$ Substrat. Zur Zeit 0 und nach 60 Min. Inkubation bei $37^{\circ}$ wurde $1 \mathrm{ml}$ entnommen, 4 Min. bei $4000 \mathrm{~g}$ zentrifugiert, der Überstand 1:1 mit 10-proz. Trichloressigsäure (TCE) enteiweißt, mit $\mathrm{NaHCO}_{3}$ neutralisiert und 1:50 mit aqua bidest. verdünnt. Hiervon wurden $0,5 \mathrm{ml}$ in die Bestimmung des Reduktionswertes nach PARK-Johnson (6) eingesetzt.

\begin{tabular}{|c|c|c|}
\hline \multirow[t]{2}{*}{ Substrat } & \multicolumn{2}{|c|}{$\begin{array}{l}\text { Reduktionswert im Überstand } \\
\text { als GA-Äquivalente }[\mathrm{mg} / \mathrm{m} l]\end{array}$} \\
\hline & 0 Minuten & 60 Minuten \\
\hline 0 & 0,00 & 0,00 \\
\hline GA & 1,75 & 0,85 \\
\hline$(G A)_{2}$ & 0,83 & 0,91 \\
\hline$(G A)_{3}$ & 0,70 & 0,79 \\
\hline$(G A)_{4}$ & 0,51 & 0,61 \\
\hline
\end{tabular}

Tab. 4

Abnahme des Glukosamingehaltes im Inkubationsmedium Versuchsbedingungen wie unter Tab. 3

$1,5 \mathrm{~m} l$ des neutralisierten TCE-Extraktes wurden mit $0,75 \mathrm{ml}$ $10 " \mathrm{HCl} 15$ Stdn. bei $105^{\circ}$ hydrolysiert und mit $\mathrm{NaOH}$ neutralisiert. Das Glukosamin wurde anschließend nach BoAs (2/4) bestimmt. Es wurde dabei auf die vorangehende Abtrennung des Glucosamins über eine Dowex 50-Säule verzichtet.

\begin{tabular}{ccc}
\hline & $\begin{array}{c}\text { Abnahme des Glukosamingehaltes } \\
\text { mg/ml }\end{array}$ \\
\hline GA & 1,04 & 52 \\
$(\mathrm{GA})_{2}$ & 0,26 & 13 \\
$(\mathrm{GA})_{3}$ & 0,16 & 8 \\
\hline
\end{tabular}

Tab. 5

Spaltung von $\mathrm{N}$-acetylierten Oligosacchariden des Glukosamins. In $2,5 \mathrm{~m} l$ Krebs-Ringer-Bikarbonat-Lösung waren $230 \mu l$ Zellen und jeweils $5 \mathrm{mg}$ Substrat enthalten. Sonstige Versuchsbedingungen s. Tab. 3).

\begin{tabular}{ccc}
\hline Substrat & $\begin{array}{c}\text { Reduktionswert im Uberstand } \\
\text { als N-acetyl }(\mathrm{GA}) \text {-Äquivalente } \\
(\mathrm{mg} / \mathrm{ml})\end{array}$ \\
& 0 Minuten & 60 Minuten \\
\hline 0 & 0,00 & 0,00 \\
$\mathrm{~N}$-acetyl (GA) & 1,03 & 1,03 \\
$\mathrm{~N}$-acetyl (GA) & 0,74 & 0,74 \\
$\mathrm{~N}$-acetyl $(\mathrm{GA})_{4}$ & 0,51 & 0,52 \\
\hline
\end{tabular}

Stoffwechsel-indifferenten acetylierten Saccharide dagegen zeigen keine meßbare Zunahme des Reduktionswertes wie Tabelle 5 zeigt. Zum empfindlicheren Nachweis wurde T-markiertes $\mathrm{N}$-acetyl-(GA) ${ }_{3}$ eingesetzt. Es konnte damit nachgewiesen werden, daß die acetylierten Verbindungen im Gegensatz zu den nicht-acetylierten während der Versuchszeit nicht gespalten werden (s. Abb. 2). Bei einer Spaltung hätten das markierte Mono- $\left(R_{\mathrm{F}}=0,80\right)$ und Disaccharid $\left(R_{\mathrm{F}}=0,50\right)$ nachweisbar sein müssen. Ebenfalls war in Übereinstimmung mit den Aussagen des Reduktionswertes, kein Trisaccharid im Zellextrakt nachweisbar.

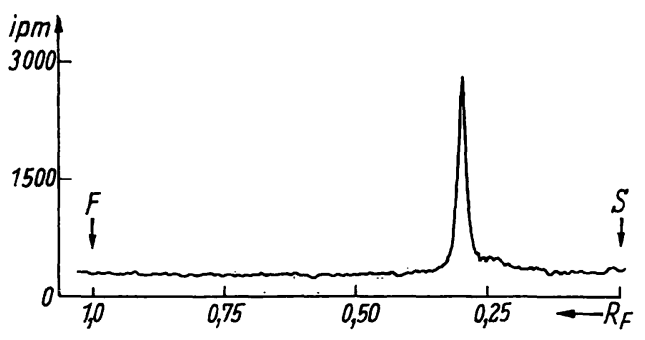

Abb. 2

Radiochromatogramm von $\operatorname{Tri}(\mathrm{N}$-acetyl)-Chitotriose-[T] nach Inkubation mit Ascitestumorzellen

$1,1 \mathrm{ml}$ einer 20-proz. Zellsuspension wird in Krebs-RingerBikarbonat-Lösung mit $0,5 \mathrm{mg}$ markiertem Trisaccharid $(20 \mu \mathrm{c})$ bei $37^{\circ}$ inkubiert. Nach einer Stunde wird abzentrifugiert und $0,1 \mathrm{~m} /$ des Überstandes auf Schleicher und Schüll-Papier 2043b dreifach chromatographiert.

\section{Intrazelluläre Phosphat-Konzentration nach Zugabe verschie- dener Substrate}

Da verschiedene Autoren die Hemmung der endogenen Atmung durch phosphorylierbare Monosaccharide durch die Abnahme der Phosphatkonzentration in den Zellen erklären (s. auch 12a), haben wir das Verhalten des Phosphatgehaltes der Zellen bei verschiedenen extrazellulären Phosphatkonzentrationen untersucht, und $z$ wàr in inaktiviertem Ascitesserum $\left(2,4 \mathrm{mMol} \mathrm{PO}_{4} / l\right)$ sowie in Krebs-Ringer-Hydrogenkarbonat bei 0 und in Krebs-Ringer-Phosphat bei $12 \mathrm{mMol} \mathrm{PO}_{4} / l$. Tabelle 6 zeigt, daß der Phosphatgehalt in Ubereinstimmung mit den Befunden anderer Autoren $(12,13,14)$ durch

Tab. 6

Intrazellulärer Phosphat-Gehalt von Ascitestumorzellen in ( $\mu \mathrm{Mol} / \mathrm{m} /$ Zellen)

\begin{tabular}{cccc}
\hline \multicolumn{4}{c}{ Inkubationsmedium } \\
Substrat & $\begin{array}{c}\text { Krebs-Ringer- } \\
\text { Bikarbonat } \\
\left(\mathrm{PO}_{4} \text {-frei) }\right.\end{array}$ & $\begin{array}{c}\text { inaktiviertes } \\
\text { Ascitesserum }\end{array}$ & $\begin{array}{c}\text { Krebs-Ringer- } \\
\text { Phosphat }\end{array}$ \\
\hline $\begin{array}{c}\text { Versuclssbeginn } \\
\text { ohne Substrat }\end{array}$ & 9,3 & 8,3 & 10,7 \\
Versuclssende & & & \\
ohne Substrat & 11,1 & 11,3 & 15,4 \\
G & 5,9 & 9,5 & 10,5 \\
GA & 0,7 & 2,1 & 6,8 \\
$(\mathrm{GA})_{2}$ & 11,0 & 10,6 & 24,8 \\
$(\mathrm{GA})_{3}$ & 11,9 & 11,1 & 22,3 \\
$(\mathrm{GA})_{4}$ & 11,4 & 10,5 & 23,2 \\
\hline
\end{tabular}


Glukose nur geringfügig gesenkt wird, wenn sich $\mathrm{PO}_{4}$ im Außenmedium befindet. Der Phosphatgehalt fällt jedoch stärker, wenn kein $\mathrm{PO}_{4}$ im Außenmedium angeboten wird. Mit Glukosamin als Substrat kam es wie wir schon früher gefunden hatten (2) - zu einer erheblichen Abnahme des Phosphatgehaltes. Die größte Senkung des Phosphatgehaltes trat wieder auf, wenn das Phosphat in der Suspensionsflüssigkeit fehlte. Bei den untersuchten Oligosacchariden kam es dagegen zu keiner Abnahme des Phosphatgehaltes, obwohl der $\mathrm{PO}_{4}$-Gehalt in allen Ansätzen zum Zeitpunkt maximaler Atmungshemmung untersucht wurde.

Der Zellgehalt bei den einzelnen Versuchen lag zwischen 7,0 und $13,5 \%$. Zu Versuchsbeginn und nach einer Inkubationszeit von $30-50$ Minuten wurden 2,0 $\mathrm{m} /$ der Zellsuspension entnommen und $5 \mathrm{Min}$. bei $4000 \mathrm{~g}$ zentrifugiert. Der Überstand wurde dekantiert. Die Zentrifugengläser wurden mit Filterpapier ausgetupft. Das Zellsediment wurde mit $1,5 \mathrm{ml} 10$-proz. TCE versetzt, mit einem Glasstab verrührt, 1 Stunde bei $0^{\circ}$ stehengelassen und $10 \mathrm{Min}$. bei $2000 \mathrm{~g}$ zentrifugiert. $0,5 \mathrm{ml}$ des TCE-Extraktes wurden zur $\mathrm{PO}_{4}$-Bestimmung nach FISKE und SubBarow eingesetzt.

Protokoll:

\begin{tabular}{lcccc}
\hline Ansatz & $\begin{array}{c}\text { Zellsusp. in } \mathrm{PO}_{4^{-}} \\
\text {freier Krebs-Ringer- } \\
\text { Bikarbonat-Lsg. }\end{array}$ & $\begin{array}{c}\text { Ringer- } \\
\text { Lsg. }\end{array}$ & $\begin{array}{c}4,4 \mathrm{mg} \text { Substrat } \\
\text { in } 0,2 \mathrm{ml} l \\
\text { Ringer-Lsg. }\end{array}$ & $\begin{array}{c}\text { Inkubations- } \\
\text { dauer bei } \\
37^{\circ}\end{array}$ \\
\hline $1 \mathrm{a}$ & 2,0 & $0,2$. & - & 0 \\
1 & 2,0 & 0,2 & - & $30^{\prime}$ \\
2 & 2,0 & - & $\mathrm{G}$ & $30^{\prime}$ \\
3 & 2,0 & - & {$[\mathrm{GA}]$} & $30^{\prime}$ \\
4 & 2,0 & - & {$[\mathrm{GA}]_{2}$} & $30^{\prime}$ \\
5 & 2,0 & - & {$[\mathrm{GA}]_{3}$} & $30^{\prime}$ \\
6 & 2,0 & - & {$[\mathrm{GA}]_{4}$} & $30^{\prime}$ \\
\hline
\end{tabular}

Zellgehalt der Suspension: 8,65 Vol. \%.

$\mathrm{PO}_{4}$-Bestimnutung in 0,5 $\mathrm{ml}$ TCE-Extrakt

\begin{tabular}{lcccccc}
\hline Ansatz & $\mathrm{E}_{1}$ & $\mathrm{E}_{2}$ & $\overline{\mathrm{E}}$ & $\mu \mathrm{g} \mathrm{P}$ & $\frac{\mu \mathrm{MolPO}_{4}}{\text { Ansatz }[173 \mu l]}$ & $\frac{\mu \mathrm{MolPO}_{4}}{\mathrm{~m} l \text { Zellen }}$ \\
\hline 1a & 0,165 & 0,180 & 0,172 & 15,2 & 1,61 & 9,3 \\
1 & 0,200 & 0,210 & 0,205 & 18,0 & 1,91 & 11,1 \\
2 & 0,110 & 0,110 & 0,110 & 9,64 & 1,02 & 5,9 \\
3 & 0,010 & 0,015 & 0,013 & 1,13 & 0,12 & 0,7 \\
4 & 0,195 & 0,210 & 0,203 & 17,9 & 1,90 & 11,0 \\
5 & 0,220 & 0,225 & 0,222 & 19,5 & 2,06 & 11,9 \\
6 & 0,205 & 0,215 & 0,210 & 18,6 & 1,97 & 11,4 \\
\hline
\end{tabular}

Spaltung von inaktiver und T-markierter Hyaluronsäure

$\mathrm{Da}$ die untersuchten Saccharide $\beta$-glukosaminidisch gebunden sind, untersuchten wir, ob physiologisch vorkommende $\beta$-hexos-aminidisch gebundene Substanzen, wie Hyaluronsäure und Chondroitinschwefelsäure ebenfalls von Ascitestumorzellen gespalten werden. $A b=$ bildung 3 zeigt einen derartigen Versuch. Wie aus dem Verhalten der Reduktionswerte ersichtlich ist, wird Hyaluronsäure von Asçitestumorzellen gespalten: $\mathrm{Ob}$ diє geringfügige Zunahme des Reduktionswertes mit Chondroitinschwefelsäure als Substrat auf einer Spaltung dieser Substanz beruht, läßt sich nicht sicher entscheiden, da bei der Inkubation von Ascitestumorzellen ohne Substrat der Reduktionswert im Medium ebenfalls etwas ansteigt.
Die pH-Abhängigkeit der Hyaluronsäurespaltung wurde mit einem Aceton-Trockenpulver (15) aus Ascitestumorzellen untersucht. Wie Abbildung 4 zeigt, liegt das pH-Optimum bei $\mathrm{pH}=5,5$.

Lim auch auf andere Weise die Hyaluronsäure-Spaltung durch Ascitestumorzellen zu bestätigen, inkubierten wir nar.h Wilzbach mit Tritium markierte Hyaluronsäure mit Asciteszellentrockenpulver. Nach 6 und 24 Stunden wurde ein aliquoter Teil entnommen, mit Trichloressigsäure enteiweißt und $6 \mathrm{mal}$ aufsteigend chromatographiert, um evtl. gleichzeitig auftretende niedermolekulare Spaltprodukte nachweisen zu können. Das Ergebnis ist in Abbildung 5 dargestellt. Nach 6 Stunden, deutlicher nach 24 Stunden zeigte sich oberhalb des Startpunktes ein zweites Aktivitätsmaximum, das durch niederpolymere Hyaluronsäure bedingt ist. Niederpolymere Oligosaccharide sowie die monomeren Bausteine

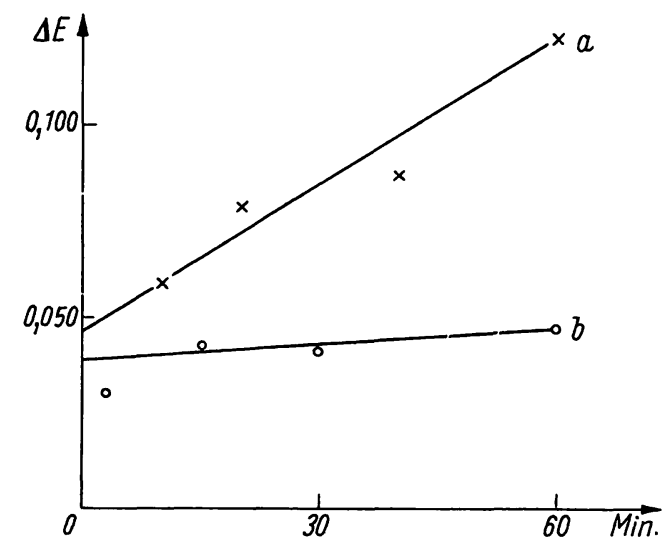

Abb. 3

Spaltung von Hyaluronsäure und Chondroitinschwefelsäure durch Ascitestumorzellen. a) Reduktionswert des Hyaluronsäureansatzes; b) Reduktionswert des Ansatzes mit Chondroitinschwefelsäure. $6 \mathrm{ml}$ einer 10-proz. Zellsuspension in Krebs-Ringer-PhosphatLösung $\mathrm{pH}=7,4$ enthielten $12 \mathrm{mg}$ Hyaluronsäure bzw. $12 \mathrm{mg}$ Chondroitinschwefelsäure. $\mathrm{Zu}$ verschiedenen Zeiten wurde jeweils $1 \mathrm{ml}$ entnommen und 5 Min. bei $4000 \mathrm{~g}$ zentrifugiert. In $0,1 \mathrm{ml}$ des Uberstandes wurde der Reduktionswert bestimmt.

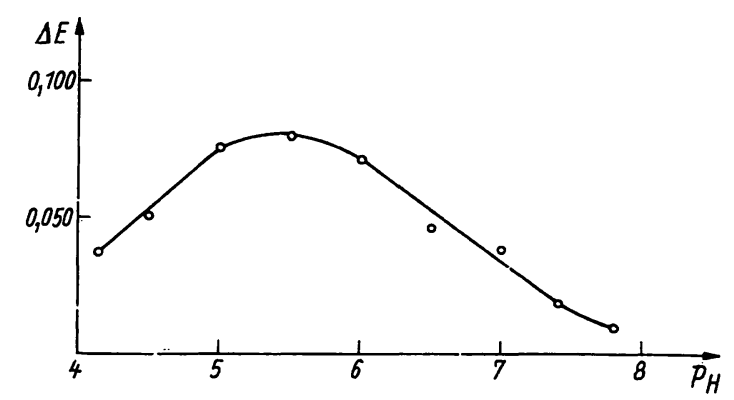

Abb. 4

pH-Abhängigkeit der Hyaluronsäurespaltung (Mittelwert aus 3 Versuchen).

Ansatz: $1 \mathrm{~m} /$ Barbital-Na-Acetat Puffer $(221)+1 \mathrm{~m} l$ AscitesTrockenpulver-Suspension ( $30 \mathrm{mg}$ in $1 \mathrm{ml} 0,9-$ proz. $\mathrm{NaCl}$ ) + $0,5 \mathrm{ml}$ Hyaluronsäurclösung ( $3 \mathrm{mg}$ in $1 \mathrm{~m} / 0,9-\mathrm{proz} . \mathrm{NaCl}$ ). Am Anfang und am Ende der Inkubation (1 Std.) wurde $1 \mathrm{ml}$ des Ansatzes entnommen und mit $1 \mathrm{ml} 10$-proz. Trichloressigsäure enteiweißt. Der TCE-Extrakt wurde mit $\mathrm{NaHCO}_{3}$ neutralisiert und davon $0,1 \mathrm{~m} l$ zur Reduktionswertbestimmung (6) verwendet. $-\Delta \mathrm{E}=0,100$ entspricht $1,25 \mu \mathrm{g} \mathrm{GA}$. 

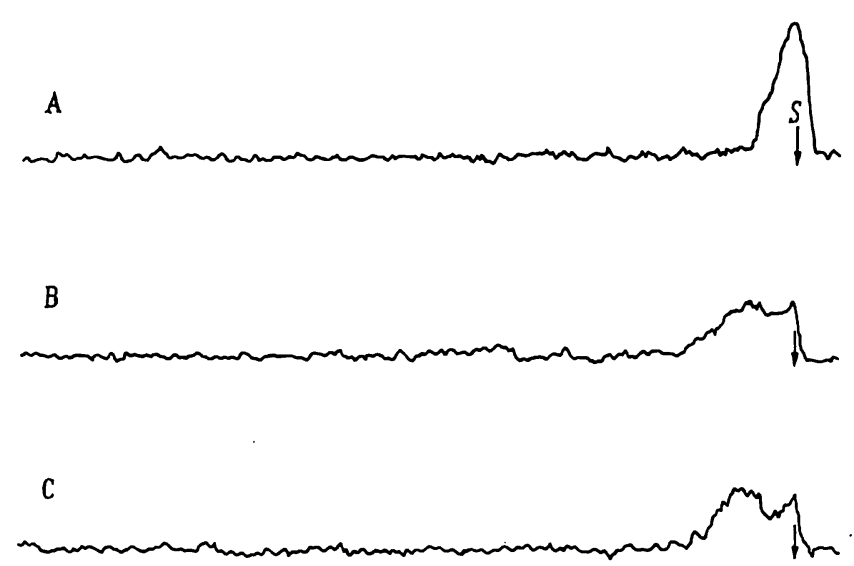

Abb. 5

Spaltung von Hyaluronsäure-[T] durch Ascitestumorzellentrockenpulver

A) Kontrolle (ohne Trockenpulver)

B) nach 6 Stunden

C) nach 24 Stunden

$1,5 \mathrm{~m} /$ 6-proz. homogenisierte Trockenpulversuspension in Barbital-Na-Acetat Puffer $(\mathrm{pH}=5,5)$ wird mit $7,5 \mathrm{mg}$ Hyaluronsäure[T] $(3,4 \mu \mathrm{c})$ bei $37^{\circ} \mathrm{im}$ Warburgapparat unter Toluolzusatz inkubiert. - Nach 6 bzw. 24 Stunden wird zu jeweils $0,5 \mathrm{~m} l$ des Ansatzes $0,1 \mathrm{ml} 30$-proz. TCE zugefügt und abzentrifugiert. $0,15 \mathrm{ml}$ des Überstandes werden auf Schleicher und Schüll Papier $2043 \mathrm{~b}$ aufgetragen und 6 -fach chromatographiert ( $\mathrm{S}=$ Start).

waren nicht nachzuweisen. Damit übereinstimmend fanden wir, daß Hyaluronsäure die Atmung und Gärung von Ascitestumorzellen niclst beeinflußt, was beim Auftreten von monomeren Glukosamin der Fall sein müßte.

\section{Diskussion}

Die Ergebnisse zeigen, daß nur phosphorylierbare Monosaccharide die endogene Atmung hemmen. Acetylierte $(\mathrm{GA})_{2},(\mathrm{GA})_{3}$ und $(\mathrm{GA})_{4}$ werden nicht gespalten, acetyliertes Glukosamin selbst wird nicht resorbiert und zeigt deswegen keinen Effekt. $(G A)_{2},(G A)_{3}$ und $(G A)_{4}$ dagegen werden gespalten; GA gelangt in die Zelle, wird hier phosphoryliert und hemmt die endogene Atmung. Gegenüber der Hemmung der endogenen Atmung durch Glukose bestehen aber gewisse Unterschiede:

Mit Glukosamin als Substrat erfolgt eine viel ausgeprägtere Senkung der intrazellulären Phosphatkonzentration als mit Glukose. Die Phosphatabnahme durch Glukose kann bei hohen Phosphatkonzentrationen im Außenmedium sogar fehlen. Trotzdem wird die Atmung gehemmt.
Mit $(G A)_{2},(G A)_{3}$ und $(G A)_{4}$ als Substrat nimmt die intrazelluläre Phosphatkonzentration überhaupt nicht ab. Dieser Befund zeigt - wie auch von anderen Autoren vermutet (16) - daß die Senkung der intrazellulären Phosphatkonzentration nicht Voraussetzung ist für die Hemmung der endogenen Atmung. Hieraus erhalten andere Theorien des Crabtree-Effektes (12, 17, 17a) eine größere Bedeutung, falls man annimmt, da $\beta$ die Hemmung der endogenen Atmung durch die verschiedenen Substrate eine gemeinsame und nicht mehrere Ursachen (18) hat, die je nach Substrat verschiedene Bedeutung haben können.

Die Atmungshemmung durch $(G A)_{2},(G A)_{3}$ und $(G A)_{4}$ beruht auf dem Abspalten von Glukosamin. Dieses erfolgt aber ziemlich langsam, so daß je nach Zellkonzentration eine verschieden lange Zeit vergeht, bis die kritische Glukosaminkonzentration erreicht ist, die die Atmung hemmt. Durch Einstellen eines Gleichgewichtes zwischen Spaltung und Resorption wird eine konstante niedrige Glukosaminkonzentration im Medium aufrechterhalten. Diese niedrige Glukosaminkonzentration reicht nicht aus, um die Phosphatkonzentration innerhalb der Zelle zu senken. Limitierend ist also nicht die Phosphorylierung des GA innerhalb der Zelle, sondern die extrazelluläre Spaltung der Oligosaccharide. Hohe Zellkonzentrationen bedingen, weil das verantwortliche Enzym auf der Zellmembran zu lokalisieren ist, eine stärkere Freisetzung von Glukosamin und damit eine höhere extrazelluläre Glukosaminkonzentration. Diese höhere extrazelluläre Glukosaminkonzentration führt zu einer schnelleren GlukosaminPenetration in die Zelle, da Glukosamin nicht durch aktiven Transport, sondern durch passive Diffusion in die Zellen gelangt (19).

Dieses Enzym wäre als $\beta$-Glukosaminidase anzusprechen. Es ist nicht identisch mit Lysozym (9), da Lysozym nur die $\mathrm{N}$-acetylierten Glukosamine spaltet, während die $\beta$-Glukosaminidase der Ascitestumorzellen nur die freien Oligomeren des GA angreift. Das Enzym scheint weiterhin auch nicht identisch zu sein mit Hyaluronidase, obwohl auch Hyaluronsäure gespalten wird. Für diese Vermutung spricht, daß Chondroitinschwefelsäure nicht meßbar gespalten wird. Weiterhin ist die $\mathrm{pH}$-Abhängigkeit der Hyaluronsäurespaltung durch Ascitestumorzellen nicht identisch mit der $\mathrm{pH}$ Abhängigkeit der Gewebs- und Testes-Hyaluronidase bei vergleichbarer Ionenstärke (20). Da keine niederpolymeren Oligosaccharide als Spaltprodukte des Hyaluronsäureabbaus auftreten, könnte das Enzym eine Endo$\beta$-Glukosaminidase sein.

\section{Literatur}

1. Günther, 'TH. und H. Greiling, Hoppe-Seyler's Z. physiol. Chem. 318, 234 (1960). - 2. Günther, Th., M. Wenzel und H. Greiling, Huppe-Seyler's' Z. physiol. Chem. 326, 212 (1961). - 3. Merker, H. J., Th. Günther und H. Greiling, Zschr. Krebsforsch. 63, 497 (1960). - 4. Hano, K., S. Matsur und Y. Nishino, Privatmitteilung (Osaka, Japan), (1962). - 5. Fiske, C. H. und Y. Subbarow, J. biol. Chemistry 66, 375 (1925).
- 6. PARK, J. und W. Johnson, J. biol. Chemistry 181, 149 (1949). - 7. LeNK, H. P., M. WeNZEL und E. SChÜTte, HoppeSeyler's Z. physiol. Chem. 326, 116 (1961). - 8. BARKER, S., A. Forster, M. Stacey und J. Webber, J. chem. Soc. (London) 2218 (1958). - 9. WeNzel, M., H. P. LeNK und E. Schütte, Hoppe-Seyler's Z. physiol. Chem. 327, 13 (1961). - 10. WENzEL, M. und P. E. Schulze, Tritium-Markierung, Darstellung, Messing 
und Anwendung nach Wilzbach 'I'-markierter Verbindungen, Walter de Gruyter \& Co., Berlin (1962). - 11. RydberG, J., Acta chem. scand. 12, 399 (1958). - 12. IBSEN, K. H., E. L. CoE und R.W. McKeE, Biochim. biophysica Acta (Amstcrdam) 30, 384 (1958). - 12a. KIEsOw, L., Z. Naturforschg. 156, 293 (1960); 166, 32 (1961). - 13. Wu, R. und E. RACKER, J. biol. Chemistry 234, 1036 (1959). - 14. Kunz, W. und W. Scrimid, Z. Naturforsch. 12b, 743 (1957). - 15. UMBreIt, W. W., R. H. Burris und J. F. Stauffer, Manometric Techniques, S. 145, 304, Burgess Publishing Co., Minneapolis (1959). - 16. ChanCE, B. und B. Hess, I. biol. Chemistry 234, 2416 (1959); 234, 2421 (1959);
Hess, B. und B. Cirance, J. biol. Chemistry 236, 239 (1961). 17. Sauer, L. A., Biochem. biophysic. Res. Commun. 17, 294 (1964). - 17a. Urbahn, H., J. Schulz, U. Hartwig und E. Hofmann, Biochem. Z. 340, 522 (1964). - 18. Hofmann, E., S. KöNIG und I. BEHNE, Naturwissenschaften 49, 40 (1962). 19. Park, C. R., D. Reinwein, M. J. Henderson, E. Cadenas und H. E. Morgan, Amer. J. Med. 25, 674 (1959). - 20. Gibian, H., Hoppe-Seyler's Z. physiol. Chem. 291, 6 (1952). 21. Mrchaelis, L., Biochem. Z. 230, 139 (1931). - 22. Boas, N. F., J. biol. Chemistry 204, 553 (1953).
Dr. rer. nat. Martin Wenzel Physiologisch-chemisches Institut der Freien Universität 1 Bcrlin 33, Arnimallee 22

\title{
Zur Frage der Gefrierdenaturierung von Serumproteinen
}

\author{
Papier- und immunoelektrophoretische Untersuchungen
}

\author{
Von F. Scheiffarth, H. Götz und R. Cerny \\ Aus der Mediziniscken Universitätsklinik Erlangen-Nürnberg (Direktor: Prof. Dr. N. Henning)
}

(Der Schriftleitung zugegangen am 16. September 1964)

\begin{abstract}
An insgesamt zehn Seren von Gesunden wurden in 251 Einzelanalysen mit Hilfe der Papier- und Agarelektrophorese die Effekte nach verschiedenen Gefriermaßnahmen in der Kühltruhe geprüft. Es konnte nachgewiesen werden, da $B$ nach 12 maligem Auftauen und Wiedereinfrieren, deutlicher jedoch erst nach 20 maligem Auftauen und Wiedereinfrieren, quantitative Veränderungen der einzelnen Seren auftraten. Die Veränderungen waren allerdings nur mit immunelektrophoretischer Technik faßbar. Sie bestanden in einer relativen Verminderung von $\alpha$-Praecipitaten, $\operatorname{der} \beta$-Siderophilinfraktion, der $\gamma$-Globuline und insbesondere der $\gamma$-Subfraktionen in einzelnen Fällen mit Fehlen der $\gamma_{1-A}$ und auch der $\gamma_{1-\mathrm{M}}$ Komponenten. Auch die Albuminfraktion war in Einzelfällen vermindert. Papierelektrophoretisch zeigte sich die Tendenz zu einer Verminderung der $\beta$-Globulinfraktion, die allerdings nicht statistisch gesichert werden konnte. Oxydase- und alkalische Phosphataseaktivität wurden in allen behandelten Seren noch nachgewiesen.

In der Diskussion wird auf die Instabilität der Globuline - gegenüber Albuminen - i. S. der Gefrierdenaturierung hingewiesen, wobei, unter Berücksichtigung der einschlägigen Literatur, in besonderem Maße die Lipoproteide des Serums betroffen sind. Insgesamt zeigen jedoch die vorliegenden Ergebnisse, daß sich die Serumeiweißkörper bei Gefrierversuchen um $-20^{\circ}$ in ihrer Konstellation stabil erweisen.

251 analyses by paper- or agar-electrophoresis were performed on the serum from 10 healthy persons, in order to study the effect of freezing. After freezing and thawing 12 times alternately, there were detectable changes, but after 20 times there were quantitative changes in the individual sera. The changes were only detectable by imunoelectrophoresis and consisted of a relative decrease of $\alpha$-precipitate, the $\beta$-siderophilin fraction, the $\gamma$-globulins and especially the $\gamma$-subfractions, in some cases with loss of the $\gamma_{1-A}$ and $\gamma_{1-M}$ components. In some cases the albumin fraction showed a tendency to decrease but this was not statistically-significant. Oxidase and alkaline phosphatase activity were still present in all the treated sera.

The instability of globulins as compared with albumins is discussed in relation to denaturation by freezing, which, according to relevant literature, largely affects the serum lipoproteins. The results show, however, that the pattern of serum proteins remains stable to freezing at $-20^{\circ}$.
\end{abstract}

Eine zuverlässige Haltbarmachung von Serum war von jeher Gegenstand der verschiedensten Versuche. Neben der einfachen Aufbewahrung im Kühlschrank und neben den speziellen Verfahren zur Herstellung einer Trockensubstanz in amorpher oder kristalliner Form hat sich für Eiweißlösungen das Tiefkiiblverfabren in besonderem Maße bewährt. - Die Gefrierdenaturierung von Proteinen wird im allgemeinen auf Aggregationsund Desaggregationseffekte (1) zurückgeführt, die ihrerseits als eine Folge der Eisbildung und Erhöhung der Ionenkonzentration in der flüssig gebliebenen Rest- lösung aufzufassen sind. Dabei spielen offenbar druckmechanische Faktoren $(2,3,4)$ eine nicht unwesentliche Rolle. Gefriergeschwindigkeit sowie Häufigkeit des Gefrierens und Auftauens können ebenfalls die Gefrierdenaturierung wesentlich mitbestimmen. Sicher spielen aber auch Art und Komplexbildung der Proteinsysteme und deren Hydratation ebenso wie die gewählten Kühltemperaturen eine Rolle.

In vorliegender Arbeit sollte mit Bezug auf die uns zur Verfügung stehende Kühltruhe ${ }^{1}$ ) die Haltbarkeit 1) „BBC“-Kühltruhe, Typ „OKL 8603 A13 220VE-N“保. 